\title{
Empirical Relation of the Fine-Structure Constant with the Transference Number Concept
}

\author{
Tomofumi Miyashita \\ Miyashita Clinic, Huruno-cho, Kawachinagano, Osaka, Japan \\ Email: tom_miya@plala.or.jp
}

How to cite this paper: Miyashita, T. (2018) Empirical Relation of the Fine-Structure Constant with the Transference Number Concept. Journal of Modern Physics, 9, 2346-2353.

https://doi.org/10.4236/jmp.2018.913149

Received: October 22, 2018

Accepted: November 24, 2018

Published: November 27, 2018

Copyright $\odot 2018$ by author and Scientific Research Publishing Inc. This work is licensed under the Creative Commons Attribution International License (CC BY 4.0).

http://creativecommons.org/licenses/by/4.0/

\begin{abstract}
The fine-structure constant of $1 / 137$ is puzzling and has never been fully explained. When the interaction coefficient is $1 / 137$, the transference number should be $136 / 137$. With the transference number concept, we noticed that we must examine the constant of $1 / 136$ instead of $1 / 137$ to discover an empirical relationship in which the fine-structure constant is related to the mass ratio of electrons and quarks. Then, the physical meaning of this empirical relationship is discussed.
\end{abstract}

\section{Keywords}

Wagner's Equation, Fluctuation and Dissipation Theory, Boltzmann Distribution, Maxwell's Demon, Additional Thermodynamic Law

\section{Introduction}

Previously, we tried to explain quantum physics using classical thermodynamics [1] [2]. However, these discussions were lacking evidential support, prompting us to search for this evidence.

Solid-oxide fuel cells (SOFCs) directly convert the chemical energy of fuel gases, such as hydrogen and methane, into electrical energy. SOFCs use a solid-oxide film as the electrolyte, and oxygen ions serve as the main charge carriers. Typically, yttria-stabilized zirconia (YSZ) is used as the electrolyte material in these cells. The open-circuit voltage ( $O C V)$ of the YSZ electrolyte is equal to the Nernst voltage $\left(V_{t h}\right)$ of $1.15 \mathrm{~V}$ at $1073 \mathrm{~K}$. However, using samaria-doped ceria $(\mathrm{SDC})$ electrolytes, the $O C V$ is approximately $0.8 \mathrm{~V}$. The low $O C V$ was calculated using Wagner's equation, which is based on the chemical equilibrium 
theory. Wagner's equation [3] [4] is

$$
J_{O_{2}}=-\frac{R T}{16 F^{2} L} \int_{\ln p O_{2}^{\text {anode }}}^{\ln p O_{\text {cathode }}} \frac{\sigma_{e l} \sigma_{\text {ion }}}{\sigma_{e l}+\sigma_{\text {ion }}} \mathrm{d} \ln p O_{2}
$$

where $J_{O_{2}}$ and $\mathrm{pO}_{2}$ are the $\mathrm{O}_{2}$ flux and the $\mathrm{O}_{2}$ partial pressure, respectively; $p O_{2}^{\text {cathode }}$ and $p \mathrm{O}_{2}^{\text {anode }}$ are the $\mathrm{O}_{2}$ partial pressures at the cathode and anode, respectively; $R, T$, and $F$ are the gas constant, the absolute temperature, and Faraday's constant, respectively; $L$ is the thickness of the membrane or film; and $\sigma_{e l}$ and $\sigma_{\text {ion }}$ are the conductivities of the electrons and oxygen vacancies, respectively.

From Equations (1), Equations (2) and (3) can be deduced [5]:

$$
O C V=V_{t h}-R_{i} I_{i}
$$

where $R_{i}$ and $I_{i}$ are the ionic resistances of the electrolyte and the ionic current, respectively.

$$
O C V=\frac{R T}{4 F} \int_{\ln p O_{2}^{\text {anode }}}^{\ln p \text { cathode }} t_{\text {ion }} \mathrm{d} \ln p O_{2}
$$

Parameter $t_{\text {ion }}$ is expressed as

$$
t_{i o n}=\frac{\sigma_{i o n}}{\sigma_{e l}+\sigma_{i o n}}
$$

However, $\sigma_{e l}$ is a function of the $\mathrm{O}_{2}$ partial pressure [6]:

$$
\sigma_{e l}=\sigma_{i o n}\left(\frac{p O_{2}}{p O_{2}^{*}}\right)^{-\frac{1}{4}}
$$

where $p O_{2}^{*}$ corresponds to the oxygen partial pressure at which $t_{\text {ion }}=1 / 2$. When $t_{i o n}$ is constant in the electrolytes,

$$
O C V=t_{i o n} \times V_{t h}
$$

The low $O C V$ was thought to be due to the low value of the ionic transference number $\left(t_{\text {ion }}\right)$. However, experimentally, $I_{i}$ in Equation (2) is negligible [6] [7] [8] [9] [10]. Considering the direction of the electrical field, there are serious problems in Wagner's equation [5] [10]. Therefore, the voltage loss should be explained by other reasons.

Over the past two decades, the understanding of nonequilibrium thermodynamics has been enhanced by fluctuation and dissipation theorems such as the Jarzynski and Crooks relations [11] [12]. The autonomous Maxwell's demon concept was proposed by Jarzynski [13], and we independently discovered the equation for this concept [14]. In our equation, $t_{\text {ion }}$ remains important. In addition, we determined the empirical relationship and discussed the physical meaning of this empirical relationship.

\section{Equation for Autonomous Maxwell's Demons}

\subsection{Main Problems in Wagner's Equation}

According to Michael Faraday, the direction of the electrical field is from the 
anode to the cathode. In the 1950s, Wagner studied mixed conductors with positively and negatively charged ions. However, Wagner's equation was used for doped ceria electrolytes in which there are two negative carriers (oxygen ions and electrons). The ionic current $\left(I_{i}\right)$ and electron drift current $\left(I_{e_{-} \text {drift }}\right)$ flow from the cathode to the anode. Only the electron diffusion current $\left(I_{e_{-} \text {diffusion }}\right)$ can flow from the anode to the cathode. A schematic drawing of the directions of $I_{p} I_{e_{-} \text {drift }}$ and $I_{e_{-} \text {diffusion }}$ is presented in Figure 1. According to Weppner [15], there should be a delay for $I_{e_{-} \text {diffusion: }}$

$$
\tau=\frac{L^{2}}{\tilde{D}}
$$

where $\tau, L$, and $\tilde{D}$ are the equilibrium time, sample length, and chemical diffusion coefficient, respectively. According to Wang [16], $\tilde{D}$ is $3.2 \times 10^{-6} \mathrm{~cm}^{2} / \mathrm{s}$ at $1073 \mathrm{~K}$. Therefore, using 1-mm-thick SDC electrolytes, $\tau$ should be $52 \mathrm{~min}$ at $1073 \mathrm{~K}$. However, such a delay has been never observed during the transient process, so the existence of $I_{e_{-} \text {diffusion }}$ can be disproved [5] [10].

\subsection{Autonomous Maxwell's Demons Explanation}

We discovered the following empirical equation using SDC electrolytes [14]:

$$
O C V=V_{t h}-\frac{E_{a}}{2 e}
$$

where e is elemental charge. $E_{a}$ is the ionic activation energy, which is $0.7 \mathrm{eV}$ for SDC electrolytes. Therefore, the $O C V$ in Equation (1) is $0.80 \mathrm{~V}(=1.15 \mathrm{~V}-0.7$ $\mathrm{eV} / 2 \mathrm{e}$ ). This equation is explained in Figures 2-4. The Boltzmann distribution of oxygen ions in the electrolyte at $1073 \mathrm{~K}$ is displayed in Figure 2. The ions with energies exceeding $E_{a}$ become carriers (hopping ions). Figure 3 presents an incorrect carrier distribution. The Boltzmann distribution cannot be separated using passive filters because of the phenomenon known as "Maxwell's demon", and an accurate distribution is provided in Figure 4. The loss of Gibbs energy is illustrated in Figure 3. Equation (8) is correct, when $t_{\text {ion }}$ is zero. When $t_{\text {ion }}$ is not zero, the equation for autonomous Maxwell's demon [14] [17] is

$$
O C V=V_{t h}-\left(1-t_{i o n}\right) \times \frac{E_{a}}{2 e}
$$

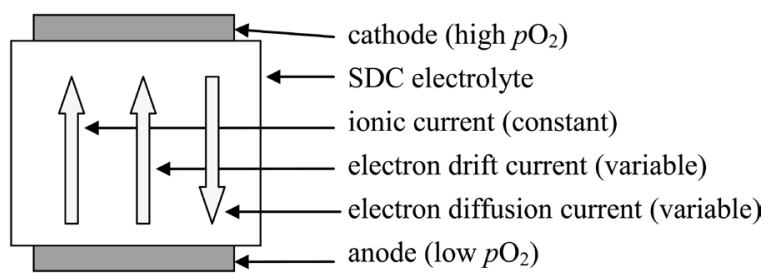

The direction of $I_{e_{-} \text {drift }}$ is the same as that of $I_{i}$.

Figure 1. Schematic drawing indicating the directions of $I_{p}, I_{e_{-} \text {drift }}$ and $I_{e_{-} \text {diffusion }}$ for the open-circuit case. 


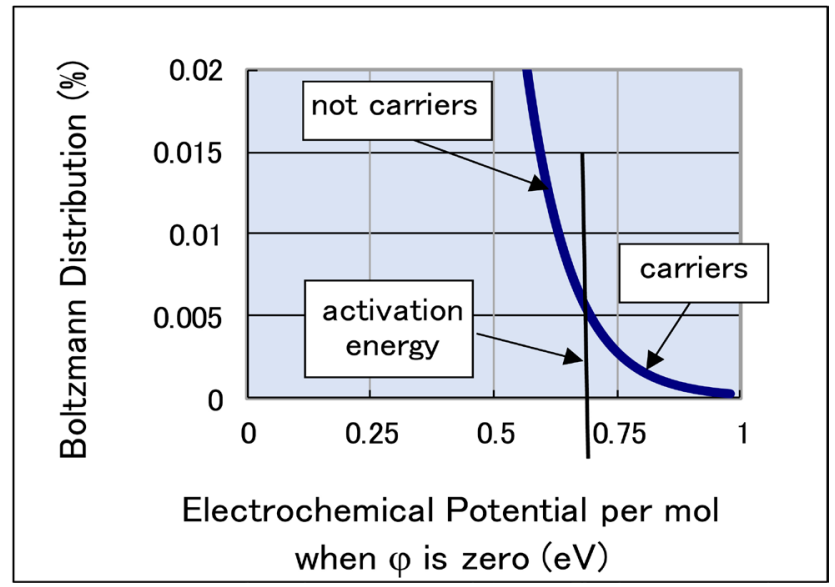

Ions with energies exceeding the ionic activation energy are converted into charge carriers (i.e., hopping ions).

Figure 2. Boltzmann distribution at $1073 \mathrm{~K}$.

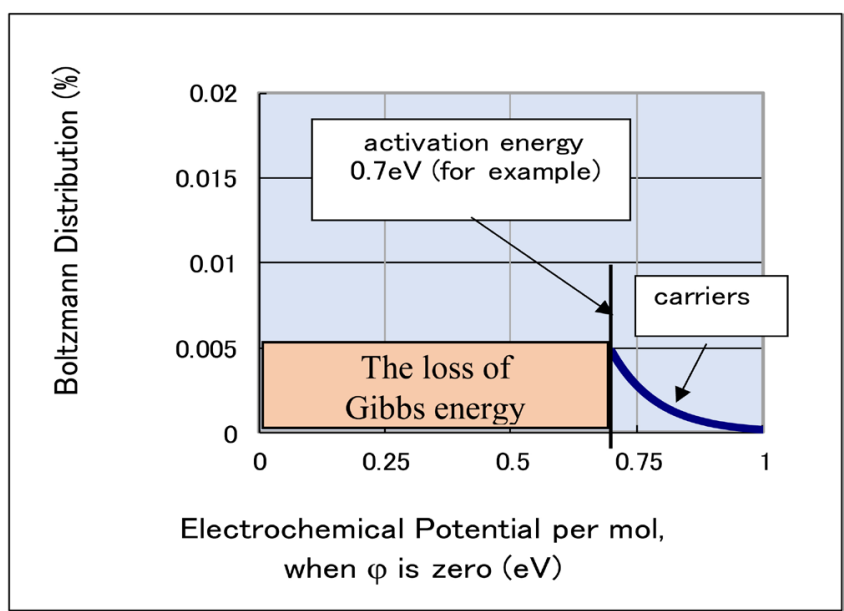

This distribution is forbidden according to Maxwell's demon.

Figure 3. Forbidden distribution of hopping ions.

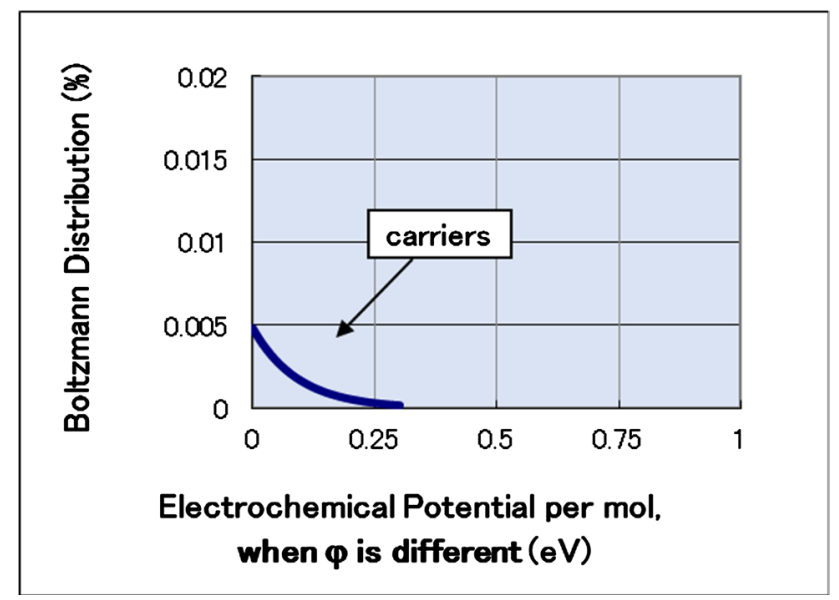

The shape of the distribution in this figure should be the same as the shape of the distribution in Figure 4.

Figure 4. Correct distribution of hopping ions. 


\section{Empirical Relations of the Fine-Structure Constant with the Transference Number Concept}

The fine-structure constant $(\alpha)$ is

$$
\alpha=\frac{e^{2}}{4 \pi \varepsilon_{0} \hbar c}
$$

where $\pi, \hbar, c$ and $\varepsilon_{0}$ are the mathematical constant pi, the reduced Planck constant, the speed of light in a vacuum and the electric constant or permittivity of free space, respectively.

$$
R_{K}=\frac{h}{e^{2}}
$$

Here, $R_{K}$ is the von Klitzing constant.

$$
Z_{0}=\frac{1}{\varepsilon_{0} c}
$$

Here, $Z_{0}$ is the characteristic impedance. Therefore,

$$
\alpha=\frac{Z_{0}}{2 R_{k}}
$$

When the interaction coefficient is $1 / 137$, the transference number should be $136 / 137$. The parameter $t_{\text {ion }}$ is expressed as

$$
t_{i o n}=\frac{\sigma_{i o n}}{\sigma_{e l}+\sigma_{i o n}}=\frac{R_{e l}}{R_{e l}+R_{i o n}}
$$

where $R_{e}$ and $R_{\text {ion }}$ are the resistance values for electrons and ions, respectively. Here, $\sigma_{\text {ion }}$ can be defined even when the ions are blocked to move. In Equation (13), we assumed that the main carriers are electrons that must move with two unknown carriers belonged to the environment. Then, the transference number unknown carriers is

$$
t_{\text {unknown }}=\frac{\frac{R_{e l}}{2}}{\frac{R_{e l}}{2}+R_{\text {unknown }}}=\frac{R_{e l}}{2\left(\frac{R_{e l}}{2}+R_{\text {unknown }}\right)}
$$

where $R_{\text {unknown }}$ is the resistance of unknown particles belonged to the environment. Equation (15) is similar to Equation (13), and $\alpha^{-1}$ is 137.035. Therefore,

$$
\frac{\frac{R_{e l}}{2}}{R_{\text {unknon }}}=136.035
$$

Next, we consider the mobility $(\mu)$ :

$$
\sigma=n e \mu
$$

where $n$ is the number of carriers.

$$
2 n_{e l}=n_{\text {unknown }}
$$

Here, $n_{e l}$ and $n_{\text {unknown }}$ are the number of electrons and the number of unknown particles, respectively. 


$$
\mu=\frac{e}{m^{*} \tau}
$$

Here, $m^{*}$ is the carrier effective mass, and $\tau$ is the average scattering time. When $\tau$ is constant,

$$
\frac{m_{\text {unknown }}}{m_{\text {el }}}=136.035
$$

where $m_{e l}$ and $m_{\text {unknown }}$ are the mass of electrons and the mass of unknown particles, respectively, and $m_{e l}$ is $0.511 \mathrm{MeV}$. Therefore, we must search for the mass with an energy value of $69.50 \mathrm{MeV}(=0.511 \times 136)$. The rest mass of a negatively charged pion has an energy of $139.57 \mathrm{MeV}$. Then, consider the following equation:

$$
m_{\pi^{-}}=2 m_{\text {quark }}+m_{e l}
$$

where $m_{\pi-}$ and $m_{\text {quark }}$ are the mass of the negatively charged pion and the mass of quarks, respectively. From Equation (21), $m_{\text {quark }}$ is $69.53 \mathrm{MeV}$, which is similar to 69.50 MeV. Therefore, our empirical equation is

$$
\frac{m_{\text {quark }}}{m_{e l}}=\frac{1}{\alpha}-1
$$

\section{Discussion}

We proposed a model in which there should be one free electron and two quarks belonged to the environment. Electrons receive the 1/137 energy of photons in the presence of an electrical field. Two quarks receive the 136/137 energy of photons. However, movement of the two quarks with the usual energy is blocked for unknown reasons. Thus, the 136/137 energy of photons should diffuse to the environment, meaning that the transference number of the space for electrons is $136 / 137$, instead of 1 , in the presence of an electrical field. We proposed that the quantity of $257,934 \mathrm{ohms}$ (from the calculation of $258,123-(377 / 2)$ ) should be measured.

When two quarks can move with higher energy, the interaction coefficient of quarks should be $136 / 137$ and the transference number of quarks should be 1/137. This is the explanation for the strong interaction. The diffusion response time of the mixed electronic and quark conductors depend exponentially on the distance. So, Yukawa potential can be explained.

\section{Conclusion}

Using the transference number concept, we proposed an empirical relationship in which the fine-structure constant is related to the mass ratio of electrons and quarks. This empirical equation is determined to be correct with a $99.96 \%$ (69.50/69.53) accuracy. Furthermore, we proposed that the quantity of 257,934 ohms should be measured. 


\section{Conflicts of Interest}

The author declares no conflicts of interest regarding the publication of this paper.

\section{References}

[1] Miyashita, T. (2011) Quantum Physics Can Be Understood in Terms of Classical Thermodynamics. Journal of Modern Physics, 2, 26-29.

https://doi.org/10.4236/jmp.2011.21005

[2] Miyashita, T. (2017) Bell's Non-Locality Theorem Can Be Understood in Terms of Classical Thermodynamics. Journal of Modern Physics, 8, 87-98.

https://doi.org/10.4236/jmp.2017.81008

[3] Wagner, C. (1975) Equations for Transport in Solid Oxides and Sulfides of Transition Metals. Progress in Solid State Chemistry, 10, 3-16. https://doi.org/10.1016/0079-6786(75)90002-3

[4] Bouwmeester, H.J.M. and Burggraaf, A.J. (1997) Dense Ceramic Membranes for Oxygen Separator. In: Gellings, P.J. and Bouwmeester, H.J.M., Eds., The CRC Handbook of Solid State Electrochemistry, CRC Press, New York, 481-553.

[5] Miyashita, T. (2017) Equilibration Process in Response to a Change in Anode Gas Using Thick Sm-Doped Ceria Electrolytes in Solid-Oxide Fuel Cells. Journal of Electrochemical Society, 164, E3190-E3199 E. https://doi.org/10.1149/2.0251711jes

[6] Kudo, T. (1997) Survey of Types of Solid Electrolytes. In: Gellings, P.J. and Bouwmeester, H.J.M., Eds., The CRC Handbook of Solid State Electrochemistry, CRC Press, New York, 195-221. https://doi.org/10.1201/9781420049305.ch6

[7] Miyashita, T. (2006) Necessity of Verification of Leakage Currents Using Sm Doped Ceria Electrolytes in SOFCs. Journal of Materials Science, 41, 3183-3184. https://doi.org/10.1007/s10853-006-6371-8

[8] Miyashita, T. (2011) Unchanged OCV Requires Concepts Considering Electrode Degradation Using Sm-Doped Ceria Electrolytes in SOFCs. Electrochemical and Solid-State Letters, 14, 1-4. https://doi.org/10.1149/1.3581208

[9] Miyashita, T. (2014) A Current-Independent Constant Anode Voltage Loss Using Sm-Doped Ceria Electrolytes in SOFCs. ECS Transactions, 59, 53-61. https://doi.org/10.1149/05901.0053ecst

[10] Miyashita, T. (2017) Current-Voltage Relationship Considering the Direction of the Electrical Field in Mixed Ionic-Electronic Solid Conductors. ECS Transactions, 80, 1057-1072. https://doi.org/10.1149/08010.1057ecst

[11] Jarzynski, C. (1997) Nonequilibrium Equality for Free Energy Differences. Physical Review Letters, 78, 2690-2693. https://doi.org/10.1103/PhysRevLett.78.2690 arXiv: cond-mat/9610209

[12] Crooks, G.E. (1999) Entropy Production Fluctuation Theorem and the Nonequilibrium Work Relation for Free Energy Differences. Physical Review E, 60, 2721-2726. https://doi.org/10.1103/PhysRevE.60.2721 arXiv:cond-mat/9901352

[13] Jarzynski, C. (2012) Work and Information Processing in a Solvable Model of Maxwell's Demon. Proceedings of National Academy of Sciences of the United States of America, 109, 11641-11645. https://doi.org/10.1073/pnas.1204263109

[14] Miyashita, T. (2005) Empirical Equation about Open Circuit Voltage in SOFC. Journal of Materials Science, 40, 6027. https://doi.org/10.1007/s10853-005-4560-5 
[15] Thangadurai, V. and Weppner, W. (2004) Ce0.8Sm0.2O1.9: Characterization of Electronic Charge Carriers and Application in Limiting Current Oxygen Sensors. Electrochimica Acta, 49, 1577-1585. https://doi.org/10.1016/j.electacta.2003.11.019

[16] Wang, S., Kobayashi, T., Dokiya, M. and Hashimoto, T. (2000) Electrical and Ionic Conductivity of Gd-Doped Ceria. Journal of Electrochemical Society, 147, 3606-3609. https://doi.org/10.1149/1.1393946

[17] Miyashita, T. (2019) Open-Circuit Voltage Anomalies in Yttria-Stabilised Zirconia and Samaria Doped Ceria Bilayered Electrolytes. (To Be Published) 